\title{
Intracranial pressure in patients with the empty sella syndrome without benign intracranial hypertension
}

\author{
ANDREW H KAYE, BRIAN M TRESS, DAVID BROWNBILL, JOHN KING
}

From the Department of Neurology and Neurosurgery, and the Department of Radiology, Royal Melbourne Hospital, Melbourne, Australia

SUMMARY The intracranial pressure was monitored continuously for at least 48 hours in five patients with empty sella syndrome, who did not have clinical benign intracranial hypertension (BIH). It has been suggested that the empty sella syndrome is a result of chronically elevated intracranial pressure in the presence of a congenitally deficient diaphragma sellae. However, whilst the intracranial pressure in two of the five patients was abnormally high, in three patients in whom it was monitored, the CSF pressure was normal. Although, these cases may represent "burnt out" forms of intracranial pressure problems, it might be that the normal pulsations of CSF are sufficient to produce the empty sella in the presence of a deficient diaphragma sellae.

\begin{abstract}
"Empty sella" is a gross descriptive term introduced by Busch in 1951 to describe the appearance at necropsy of the sella turcica when the diaphragma sellae is incomplete or forms only a small peripheral rim. ${ }^{1}$ In 1924, Schaeffer described the gross appearance of the diaphragma sellae in 125 cases, and noted that the diaphragm varied from being a complete roof which transmitted the infundibulum to, at the other extreme, a small peripheral rim..$^{2}$ In $\mathbf{4 0}$ of the 788 patients with no known pituitary disease studied by Busch (an incidence of $5.5 \%$ ), the diaphragma sellae was a peripheral rim of tissue two millimetres or less, with the pituitary gland flattened to the bottom of the sella.
\end{abstract}

The term "empty sella syndrome" originally referred to the findings at surgical exploration in patients who had received radiation for an intrasellar tumour, and subsequently developed visual symptoms. ${ }^{3}$ Primary (idiopathic) and secondary forms of the empty sella syndrome have been described. ${ }^{4}$ The primary empty sella has been defined as "that which did admit significant air at pneumoencephalography, in the absence of prior surgery or radiation therapy". ${ }^{5}$ The role of a chronically elevated cerebrospinal fluid (CSF) pressure in the pathogenesis of the so-called idiopathic empty sella syndrome has been discussed. ${ }^{6-10}$

Address for reprint requests: Andrew H Kaye, Department of Neurosurgery, Radcliffe Infirmary, Oxford OX2 6HE, UK.

Received 28 January 1981 and in revised form 13 June 1981 Accepted 25 October 1981
It has been suggested that the primary empty sella is caused by a combination of a congenitally deficient diaphragma sellae and chronically elevated CSF pressure. 51112

There is a definite relationship between benign intracranial hypertension (BIH) and the primary empty sella syndrome. ${ }^{6}{ }^{911-13}$ Although it has been suggested that there must be a chronic elevation of intracranial pressure (ICP) in addition to a deficient diaphragma sellae to produce a prolapse of the subarachnoid space into the sellae turcica, ${ }^{12}$ there has been no adequate measurement of the ICP of patients with the empty sella syndrome who do not have benign intracranial hypertension.

This is a report of a series of five patients all with radiologically proven empty sella syndrome, who did not have benign intracranial hypertension. In all patients the ICP was monitored continuously for at least 48 hours.

\section{Patients and methods}

All five patients were adult females who presented to the Royal Melbourne Hospital with a major complaint of longstanding headaches. There was no significant neurological deficit in any patient and all had normal visual fields on perimetry. A full clinical and laboratory endocrine assessment was made in all patients and all patients had a normal serum level of prolactin. No significant abnormality was found in any patient. All patients had neuroradiological investigations proving the existence of an empty sella. The diagnosis of empty sella was based on a combination of an enlarged sella with characteristic 
"symmetrical ballooning" or globular appearance on plain skull radiograph ${ }^{5}$ and the detection of CSF density within the sella by computed tomography (CT) performed with slice widths collimated to five millimetres and overlapped in both transverse axial and direct coronal planes.

The diagnosis was confirmed in one case by pneumoencephalography and another by CT metrizamide cisternography..$^{14} 15$

CT has been successful in diagnosing the presence of an empty sella in up to $100 \%$ of cases. ${ }^{14} 1617$ False positive diagnoses have been produced by prolactin secreting tumours with necrotic centres, ${ }^{1618}$ a possibility which was excluded in this series by pituitary function tests.

The ICP was monitored continuously in all patients for a minimum of 48 hours by a subarachnoid screw inserted in the right frontal position (just in front of the coronal suture). This was connected by a $150 \mathrm{~cm}$ salinefilled tube to a Hewlett Packard transducer and then to a Hewlett Packard amplifier which was connected to a continuous paper recorder. A Hewlett Packard 1208C transducer was attached to a Hewlett Packard 78200 series patient monitor which houses a 78205B amplifier. This was attached to a Hewlett Packard 7803B monitorscope and to a Rikadenki single channel recorder, modified by the Royal Melbourne Hospital Electronics Department, for two channel multiplex operation. The zero reference point for the transducer was taken as a point three centimetres anterior to the external auditory meatus, with the patient lying supine. ${ }^{19}$ The zero drift and gain drift of the system was checked, and if necessary corrected, at least every six hours. Very little correction was required at any time. Antibiotics (cloxicillin and ampicillin) were administered during the monitoring period.

Case 1 This 67-year-old obese woman, referred for investigation of the cause of two months' constant headache, had been previously reported by one of the authors ${ }^{20}$ (AHK). Neurological and general examination was normal. The plain skull radiographs revealed an enlarged globular sella (fig 1a). CT showed normal size ventricles, but also an enlarged sella with density consistent with that of CSF. A pneumoencephalogram showed air entering the anterior and mid portions of the sella, and the pituitary flattened against the dorsum and posterior portion of the floor of the sella. During the follow-up examination three months after presentation, she developed intermittent CSF rhinorrhoea. Continuous ICP monitoring revealed a generally raised baseline pressure with intermittent high plateau waves up to $34 \mathrm{~mm} \mathrm{Hg}$ (fig 2). On the basis of the elevated ICP and its presumed causal relationship with the empty sella syndrome and CSF rhinorrhoea, it was decided to perform a lumbar peritoneal shunt as an initial procedure rather than a direct intracranial attack on the fistula itself. After operation, the patient was initially free of CSF rhinorrhoea and the ICP, measured again by the above technique, demonstrated a normal baseline pressure and the elimination of wave forms. However, after 10 weeks there was a recurrence of the CSF rhinorrhoea. She was readmitted to hospital and the sella was explored through

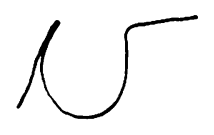

a) Case 1

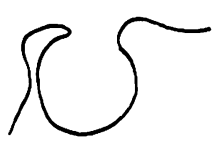

c) Case 3

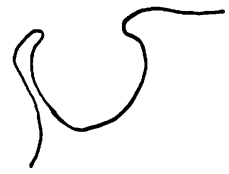

b) Case 2

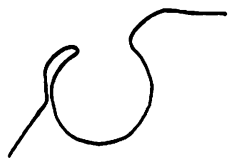

d) Case 4

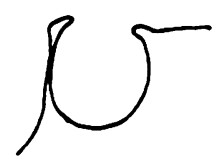

e) Case 5

Fig 1 Tracings of the sella turcicas of each of the five cases, as seen in a standard lateral skull radiograph. In all cases the volume of the sella was increased. In particular the depth was increased, being in the range 15-16 $\mathrm{mm}$ (upper level normal $14 \mathrm{~mm}$ ).

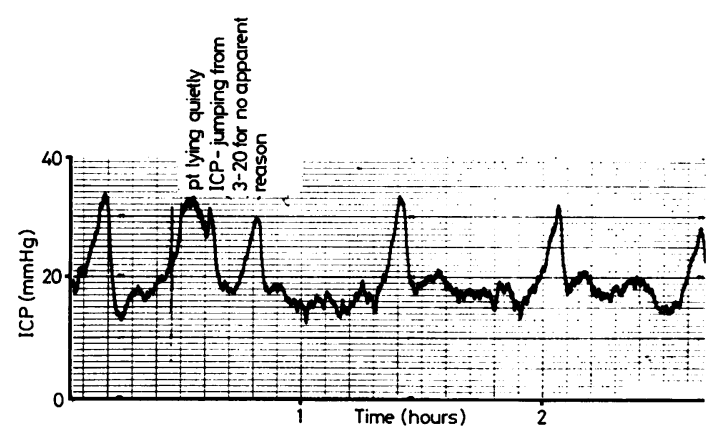

Fig 2 Intracranial pressure recording of Case 1, showing a moderately raised baseline pressure with plateau waves up to $34 \mathrm{~mm} \mathrm{Hg}$.

a right subfrontal craniotomy. At surgery an empty sella was found and a fold of arachnoid was noted to extend down into and occupy the sella. A hole, present at the junction of the floor with the anterior wall of the sella, was packed with crushed muscle and covered with fascia lata.

She has subsequently been followed for two years and has had no further CSF rhinorrhoea.

Case 2 This 70-year-old obese Italian lady presented after 12 months of moderately severe continuous headache. On examination there was no neurological abnormality and endocrine studies were normal. Lumbar puncture pressure was $12 \mathrm{~cm}$ of CSF. A plain skull radiograph showed an enlarged globular sella (fig 1b). 
CT using the above technique showed normal sized ventricles with an enlarged sella containing intrasellar attenuation values of CSF (fig 3).

The ICP monitoring for 56 hours showed a slightly elevated baseline pressure, with plateau waves rising to $30 \mathrm{~mm} \mathrm{Hg}$ and Lundberg "B" waves ${ }^{21}$ up to $35 \mathrm{~mm} \mathrm{Hg}$ (fig 4). These " $\mathrm{B}$ " waves were present during $20 \%$ of the monitoring time and there was no particular predilection for them to occur during the sleeping period.

Case 3 This 28-year-old obese female presented after some years of headaches. Neurological examination and endocrine studies were normal. Plain skull radiographs showed the characteristically enlarged globular sella (fig 1c), and CT, using the above technique, showed normal size ventricles with an enlarged sella containing CSF density material (fig 5).

The ICP was monitored for 48 hours. The baseline ICP

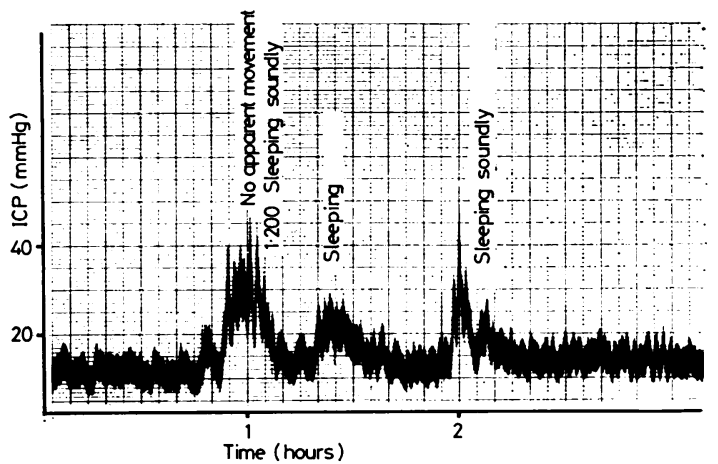

Fig 4 ICP trace of Case 2, showing a slightly elevated baseline pressure, with plateau waves rising to $30 \mathrm{~mm}$ $\mathrm{Hg}$ and Lundberg "B" waves up to $35 \mathrm{~mm} \mathrm{Hg}$.
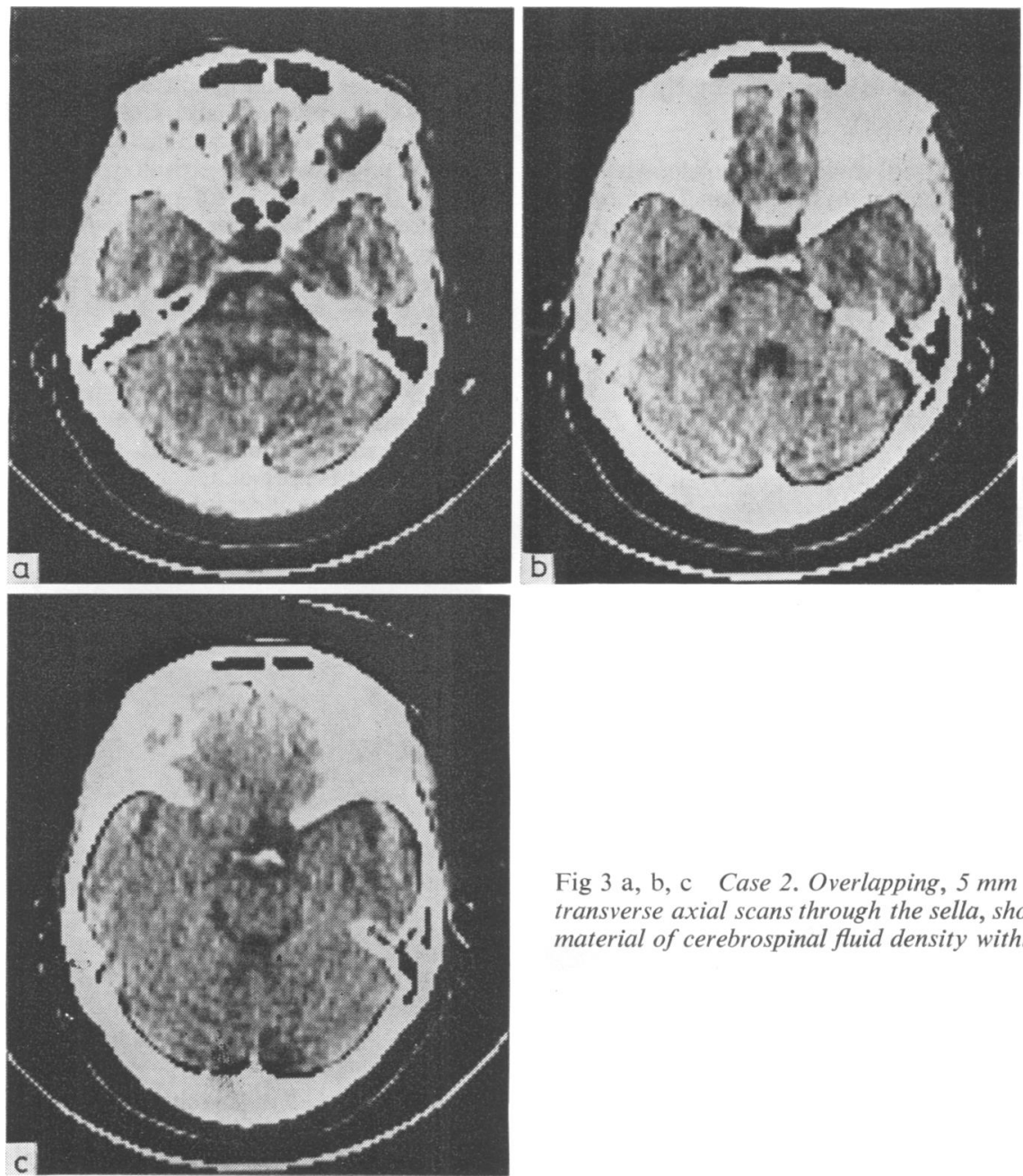

Fig 3 a, b, c Case 2. Overlapping, $5 \mathrm{~mm}$ thick transverse axial scans through the sella, showing material of cerebrospinal fluid density within it. 

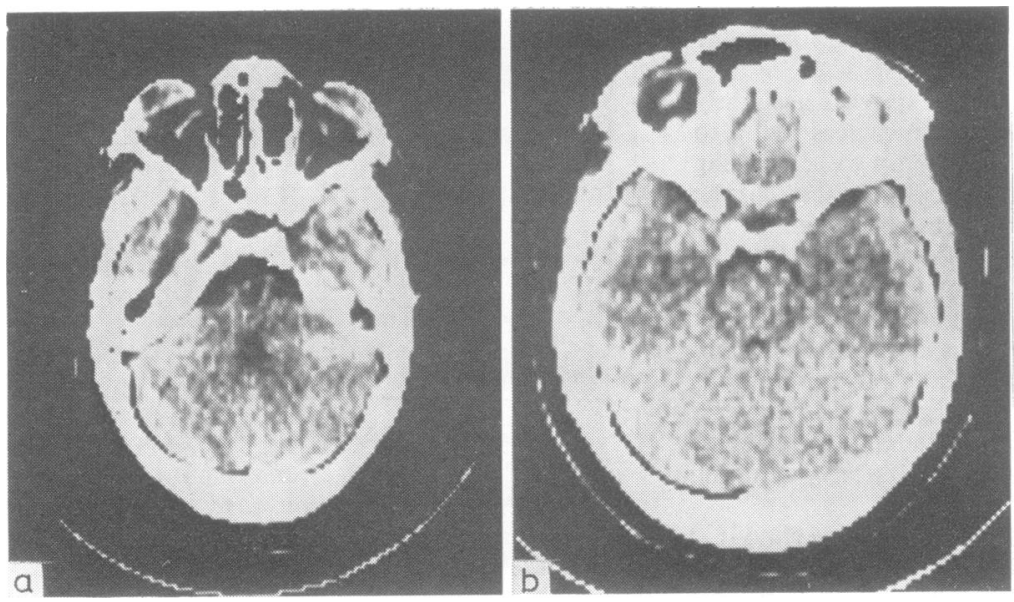

Fig 5 a, b Case 3. Two $5 \mathrm{~mm}$ thick transverse axial scans through sella turcica, showing low density contents. was within normal limits. Lundberg "B" waves occurred for approximately $5 \%$ of the monitoring time, particularly during sleep, and rose to as high as $20 \mathrm{~mm} \mathrm{Hg}$ (fig 6a and b).

Case 4 This 34-year-old rather thin female, with a past history of asthma and a hysterectomy for menstrual irregularity, presented following some years of moderately
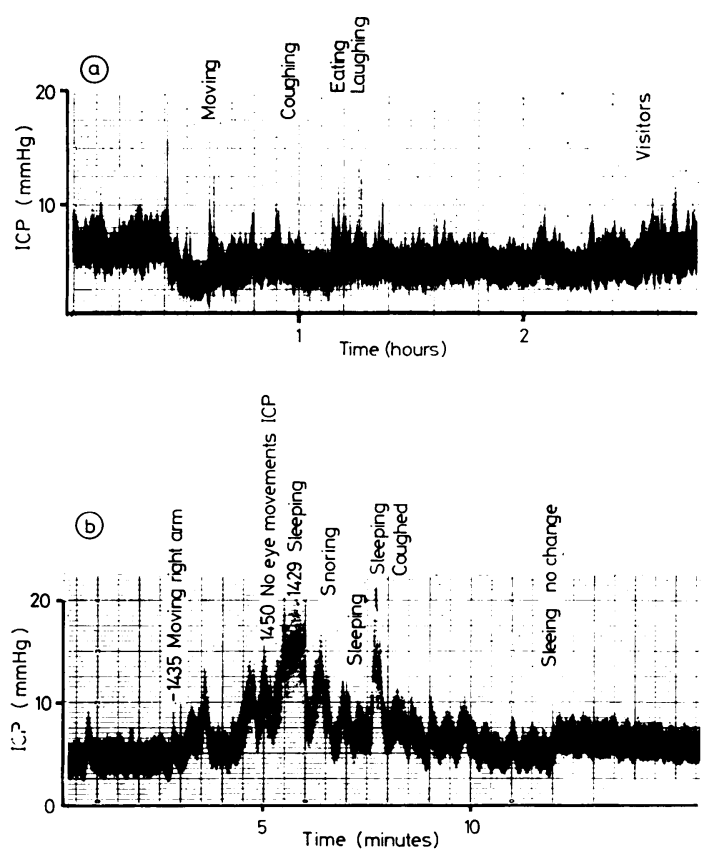

Fig 6 a, b ICP trace of Case 3, showing normal baseline pressure (a) and Lundberg " $B$ " waves occurring during sleep (b), being present for approximately $5 \%$ of the monitoring time. severe headache. There was no significant neurological abnormality and endocrine studies were normal.

A plain skull radiograph showed the characteristic features of scaphocephaly. Convolutional impressions were marked particularly in the upper third of the vault, and the sella was symmetrically enlarged in a globular fashion (figs 1d and 7). CT performed in transverse axial and direct coronal planes confirmed the presence of CSF density material in the sella (fig 8 ).

The ICP was monitored for 48 hours. The baseline ICP pressure was within normal limits and there was no significant wave formation (fig 9).

Case 5 This 41-year-old obese female presented following many years of constant headache. There was no neurological abnormality and a full endocrinological assessment was normal.

A plain skull radiograph showed an enlarged globular

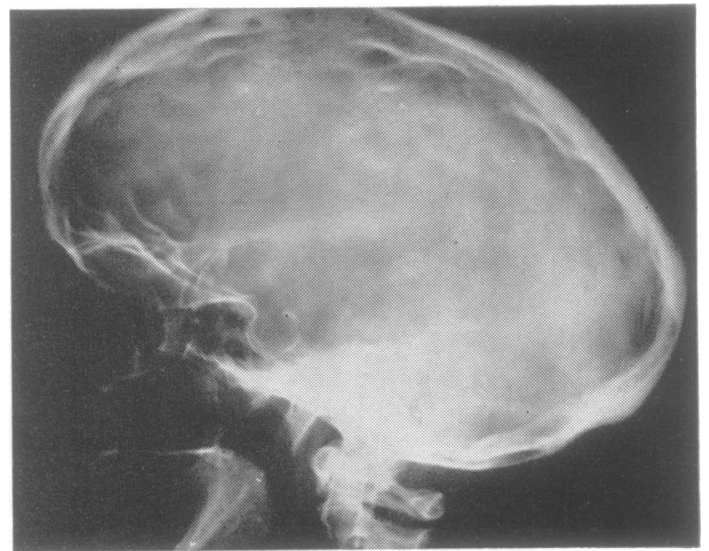

Fig 7 Plain skull radiograph of Case 4, showing marked convolutional impressions, particularly in the upper third of the vault, and an enlarged globular sella. 

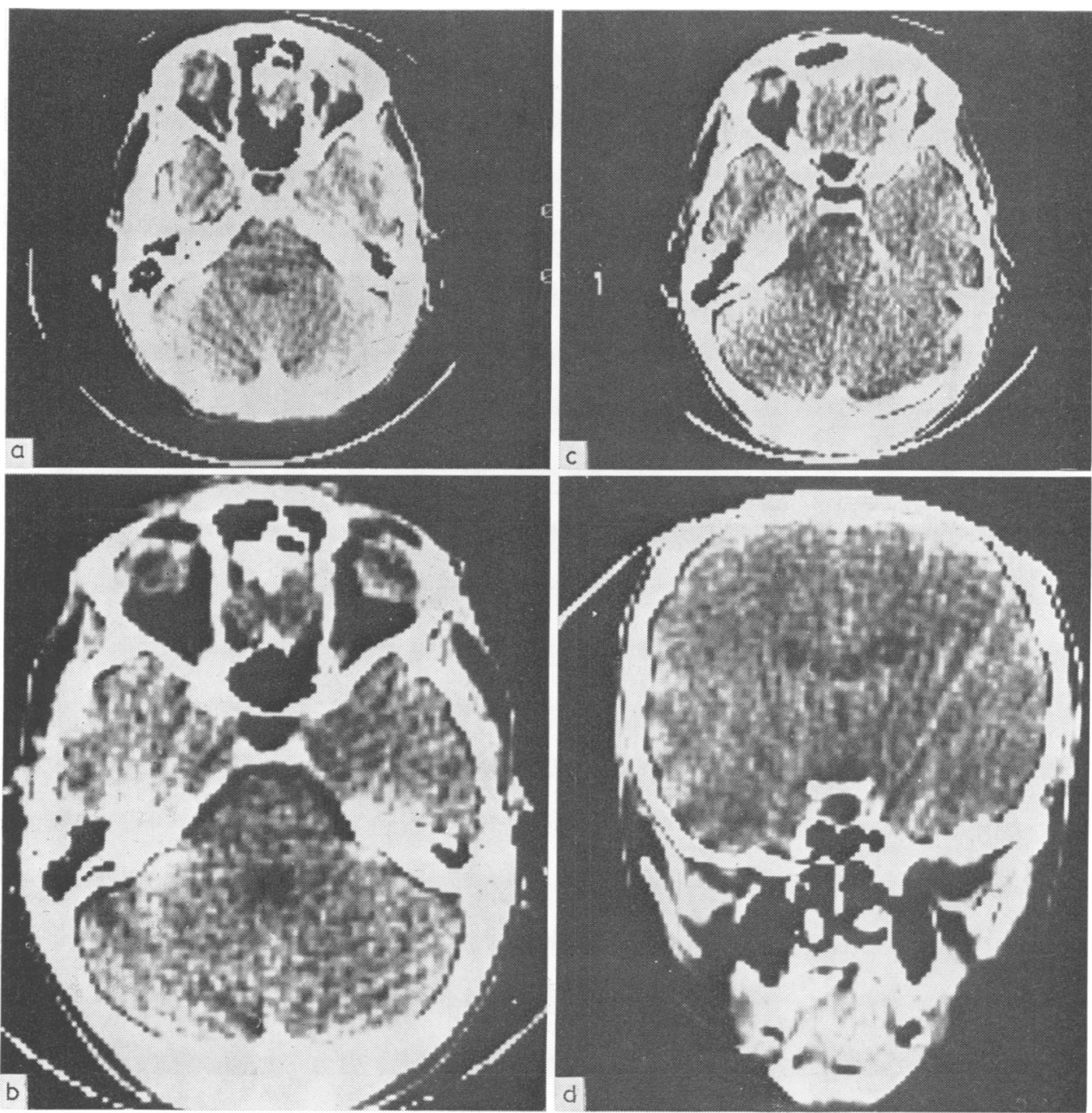

Fig 8 Case 4 (a, b, c). Overlapping 5 mm thick transverse axial scans through sella turcica and (d) $5 \mathrm{~mm}$ thick coronal slice through sella, demonstrating the sella to contain cerebrospinal fuid density material.

sella (fig 1e). CT in transverse axial and coronal planes, with and without intravenous contrast medium, showed CSF density material within the sella (fig 10). CT performed in the same planes after the introduction of intrathecal metrizamide showed a marked increase in the density of the int rasellar contents, confirming the presence of an empty sella (fig 11).

The ICP was monitored continuously for 56 hours. The baseline ICP was within normal limits and there was no significant wave formation (fig 12).

\section{Discussion}

The most commonly accepted aetiologic hypothesis for the empty sella concerns transmission of elevated

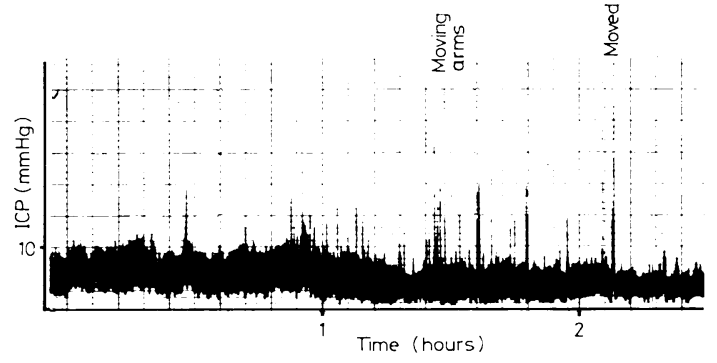

Fig 9 ICP trace of Case 4, demonstrating normal pressures. 

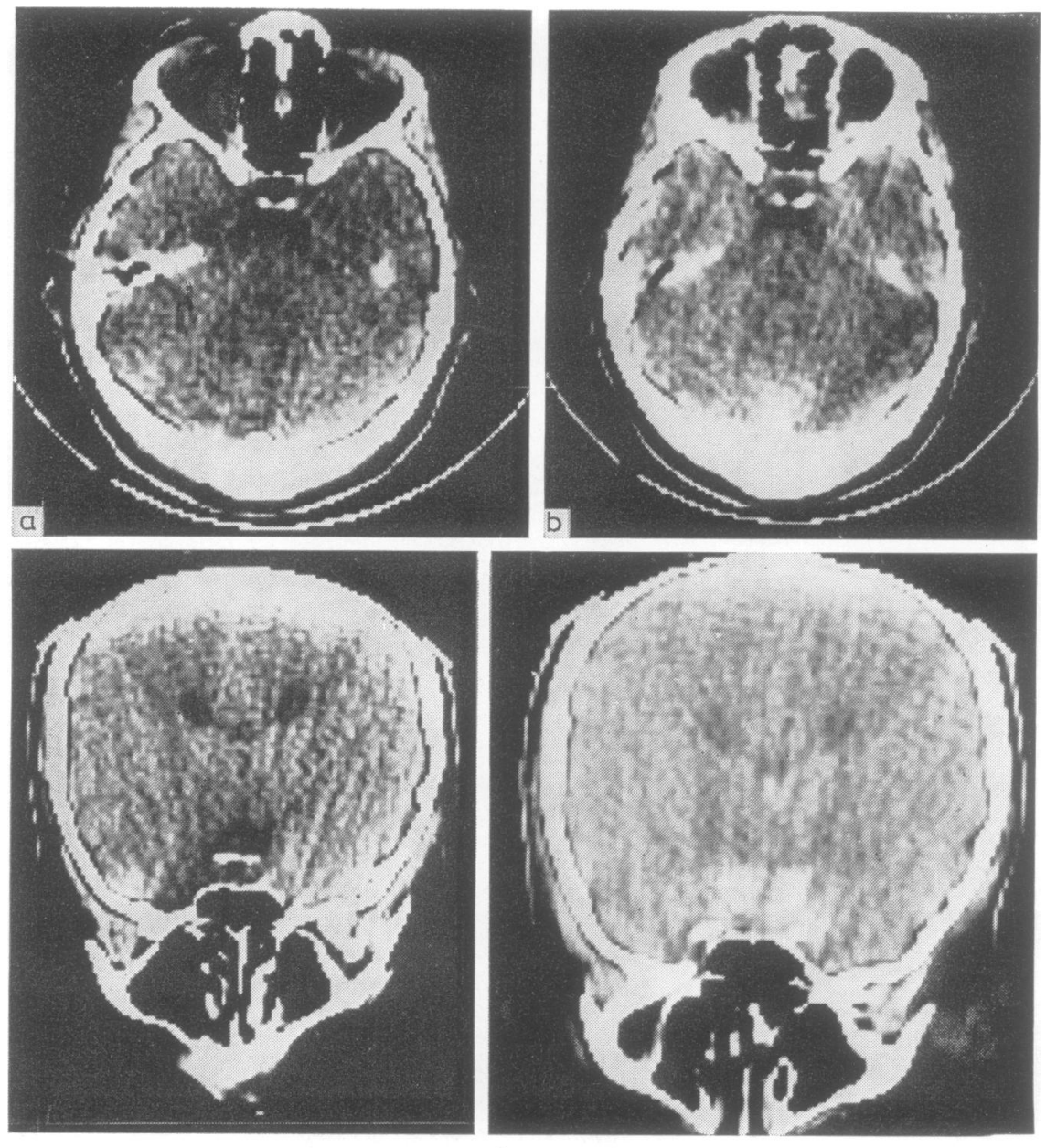

d

Fig 10 Case 5. Overlapping 5 mm thick scans in transverse axial $(\mathrm{a}, \mathrm{b})$ and coronal $(\mathrm{c}, \mathrm{d})$ planes, showing cerebrospinal fluid density material in sella.

CSF pressure through a congenitally deficient diaphragma sellae. 51112 There is a definite relationship between benign intracranial hypertension and development of the empty sella syndrome. ${ }^{611-13}$ However, those patients with benign intracranial hypertension form a minority in any series of patients with empty sella syndrome, ranging from $8 \%$ of cases, ${ }^{12}$ to $13 \%{ }^{5}$ and $15 \cdot 8 \%{ }^{6}$ Normal CSF pressure has been recorded in patients with empty sella.51011 22 However, in no study was continuous ICP monitoring performed. The importance of continuous versus static measurements of ICP has been shown in normal pressure hydrocephalus, where intermittent high pressure peaks have been demonstrated. ${ }^{23}$ Neelon argues that longstanding mild elevation of CSF pressure in the setting of incomplete sella diaphragm can bring about intru- sion of the subarachnoid space into the sella cavity with enlargement and remodelling of the sella turcica and flattening of the pituitary contents against the floor. 5

However, intermittent raised ICP cannot be adequately evaluated without using continuous ICP monitoring.

In this series of five patients with empty sella, none of whom had benign intracranial hypertension on clinical grounds and in whom continuous ICP recordings had been performed over at least a 48 hour period, a spectrum of ICP was seen from the definitely abnormal, as in the patient (Case 1) who had marked wave formation, to those patients (Cases 3, 4 and 5) who had normal ICP. Although some Lundberg "B" wave activity has been reported as being normal, ${ }^{24}$ that seen in Case 2 was probably 

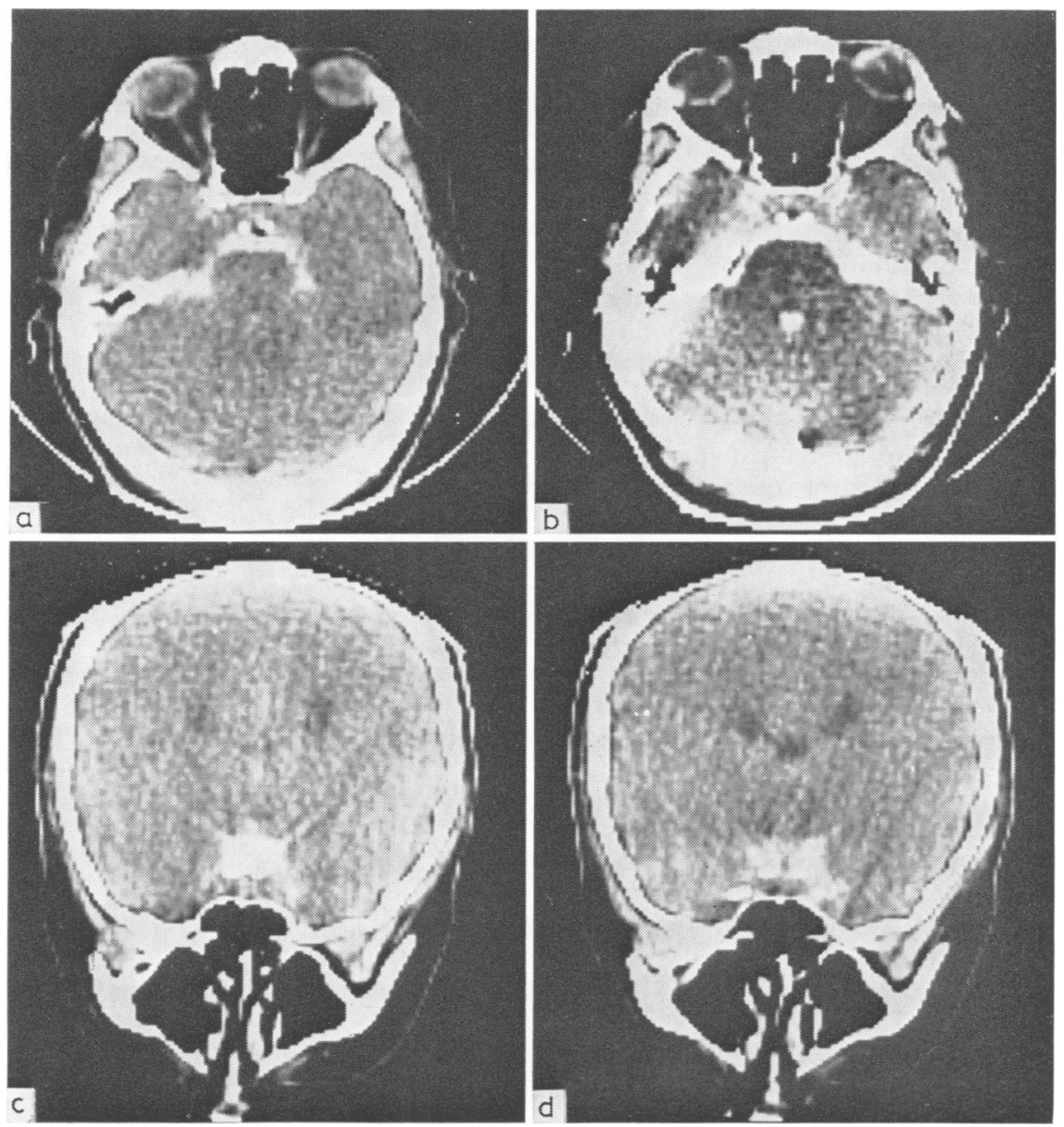

Fig 11 Case 5. After introduction of intrathecal metrizamide the density of the sella contents is markedly increased, shown in both transverse axial $(\mathrm{a}, \mathrm{b})$ and coronal $(\mathrm{c}, \mathrm{d})$ planes.

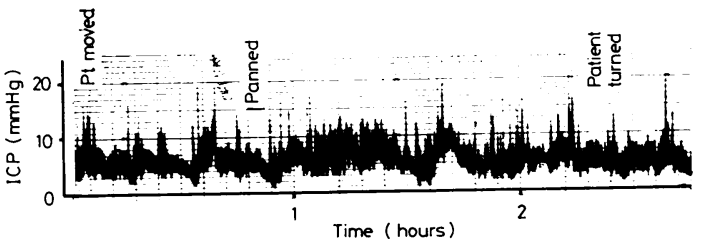

Fig 12 ICP time of Case 5, showing normal pressures.

abnormal, in that it occurred for a large percentage of the monitoring time with a high amplitude. With the exception of Case $1,{ }^{20}$ ICP recordings have not been previously reported in patients with empty sella syndrome who do not have benign intracranial hypertension. The recordings in those patients whose ICP is raised (Cases 1 and 2), help in the understanding of aetiology of the empty sella syndrome. Benign intracranial hypertension has been described in the absence of papilloedema, ${ }^{25-27}$ and Cases 1 and 2 may well represent examples of either benign intracranial hypertension without papilloedema or a "forme fruste". The ICP traces in both cases are comparable not only with those reported by Johnstone and Paterson, ${ }^{28}$ in patients with papilloedema and benign intracranial hypertension, but also with those cases of Spence et al, ${ }^{27}$ having the disorder without papilloedema. Alternatively it has been suggested that obesity itself may cause a chronic elevation of 
ICP. 511 Whatever the cause of the pressure waves, the resulting raised ICP may be sufficient to prolapse the arachnoid into the sella.

The normal ICP found in Cases 3, 4 and 5 does not necessarily negate the hypothesis of raised ICP contributing in the aetiology of empty sella syndrome. The headache occurring in these patients is not readily explainable. In the patient (Case 4) who had a scaphocephalic skull with mạked copper beating, it is interesting to speculate on the relevance of the convolutional markings in the presence of the empty sella syndrome, and at the present time normal ICP. These skull radiograph changes may represent evidence of previously raised ICP. The ICP levels of the other two normal patients (Cases 3 and 5) may represent "burnt out" intracranial hypertension. That is, those patients with normal ICP have had at some time in the development of the empty sella a high CSF pressure. Alternatively, the concept of the raised ICP being necessary for the development of the empty sella syndrome may have to be reconsidered. It may be that the normal pulsations of $\mathrm{CSF}^{29}$ are sufficient in the presence of an incomplete diaphragma sellae, to produce in some patients the empty sella syndrome.

The authors thank Mr David Wallace, Assistant Neurosurgeon, Royal Melbourne Hospital for permission to report Case 5. This work was undertaken with help from a grant from the Victor Hurley Medical Foundation.

\section{References}

1 Busch W. Die morphologia der sella turcica and ihr beziehungen zur hypophyse. Virchows Arch Pathol Anat 1951;320:437-58.

${ }^{2}$ Schaeffer JP. Some points in the regional anatomy of the optic pathways with especial reference to tumours of hypophysis cerebri and resulting ocular changes. Anat Rec 1924;28:243-79.

${ }^{3}$ Colby MY, Kearns TP. Radiation therapy of pituitary adenomas with associated visual impairment. Mayo Clin Proc 1962;37:15-24.

4 Weiss SR, Raskind R. Non neoplastic intrasellar cysts. Inter Surg 1969;51:282-6.

${ }^{5}$ Neelon FA, Goree JA, Lebovitz HE. The primary empty sella. Clinical and radiographic characteristics and endocrine function. Medicine 1973;52:73-92.

' Berke JP, Buxton LF, Kokmen E. The "empty" sella. Neurology 1975;25:1137-43.

7 Garcia-Uria J, Carrillo R, Serrano P et al. Empty sella and rhinorrhoea. $J$ Neurosurg 1979;50:466-71.

${ }^{8}$ Mortara $\mathbf{R}$, Norrell $\mathbf{H}$. Consequences of a deficient sella diaphragma. $J$ Neurosurg 1970;32:565-73.

- Weisberg LA, Zimmerman EA, Frantz AG. Diagnosis and evaluation of patients with an enlarged sella turcica. Am J Med 1976;61:590-6.

10 Zatz LM, Janon EA, Newton TH. The enlarged sella and the intrasellar cistern. Radiology 1969;93:108591.

11 Kaufman B. The "empty" sella turcica-a manifestation of the intrasellar subarachnoid space. Radiology 1968;90:931-41.

${ }^{12}$ Foley KM, Posner JB. Does pseudotumour cerebri cause the empty sella syndrome? Neurology (Minneap) 1975;25:565-9.

${ }^{13}$ Davis S, Tress B, King J. Primary empty sella syndrome and benign intracranial hypertension. Clinical and experimental neurology. Proc Aust Assoc Neurol $1978 ; 15: 248-57$.

14 Bajraktari X, Bergstrom M, Brismar K, Goulatia R, Greitz T, Grepe A. Diagnosis of intrasellar cisternal herniation (empty sella) by computer assisted tomography. J Comput Assist Tomogr 1977;1:105-16.

15 Manelfe C, Pasquini V, Banks WO. Metrizamide demonstration of the subarachnoid space surrounding the optic nerve. J Comput Assist Tomogr 1978; 215:545-7.

${ }^{16}$ Rozaria R, Hammerschlag SB, Post KD, Wolpert SM, Jackson I. Diagnosis of empty sella with C.T. scan. Neuroradiology 1977;13:85-8.

${ }^{17}$ Sage MR, Chan ES, Reilly PL. The clinical and radiological features of the empty sella syndrome. Clin Radiol 1980;31:513-9.

${ }^{18} \mathrm{Hsu}$ TH, Shapiro JR. Hyperprolactinaemia associated with empty sella syndrome. JAMA 1979;235:2002-4.

19 Azevedo Filho HRC. Clinical applications of intracranial pressure monitoring. Oxford University, 1975;76-7. (MSc thesis.)

${ }^{20}$ Davis S, Kaye AH. A dynamic pressure study of spontaneous CSF rhinorrhoea in the empty sella syndrome. $J$ Neurosurg 1980;52:103-5.

${ }^{21}$ Lundberg $N$. Continuous recording and control of intracranial pressure in neurosurgical practice. Acta Psychiatr Neurolog Scand 1960;36:(suppl 149): 1-193.

22 Bernasconi V, Giovanelli MA, Papo I. Primary empty sella. $J$ Neurosurg 1972;36:157-61.

${ }^{23}$ Symon L, Dorsch NWC, Stephens RJ. Pressure waves in so called low pressure hydrocephalus. Lancet 1972;2:291-2.

${ }^{24}$ Martin G. Lundberg's B waves as a feature of normal intracranial pressure. Surg Neurol 1978;9(6):347-8.

${ }^{25}$ Cooper PR, Moody S, Skear F. Chronic monitoring of intracranial pressure using an in vivo calibrating sensor. Experience in patients with pseudotumour cerebri. Neurosurgery 1979;5:666-70.

${ }^{26}$ Scanarini M, Mingrino S, d'Avella D, Della Corte V. Benign intracranial hypertension without papilloedema. Case report. Neurosurgery 1979;5:376-7.

27 Spence JD, Amacher AL, Willis NR. Benign intracranial hypertension without papilloedema. Role of 24 hours cerebro spinal fluid pressure monitoring in diagnosis and management. Neurosurgery 1980; 7(4):326-36.

28 Johnstone I, Paterson A. Benign intracranial hypertension II CSF pressure and circulation. Brain 1974; 97:301-12.

${ }^{29} \mathrm{Du}$ Bourlay GH. Pulsatile movements of CSF pathways. Br J Radiol 1966;39:255-62. 


\section{Correction}

In the article "Intracranial pressure in patients with the empty sella syndrome without benign intracranial hypertension" Kaye, Tress, Brownbill, King, J Neurol Neurosurg Psychiatry 1981;45:209-16 figure $10(\mathrm{~d})$ was incorrect. The correct figure $10(d)$ is reproduced here.

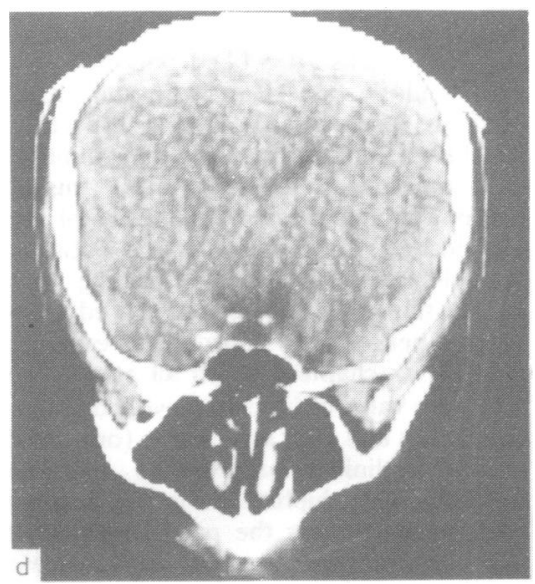

\section{Book reviews}

Psychotropic Drugs. Plasma Concentration and Clinical Response. Edited by Graham D Burrows and Trevor $\mathbf{R}$ Norman. (Pp 544; SFr. 150.) New York: Marcel Dekker Inc, 1981.

Correlation of drug effect with plasma levels is of use in many disease states. One of the most difficult areas, however, is that of psychiatric illness. The variety of means of assessing or classifying mental illness, the poor understanding of underlying pathology and the changes in brain function that occur, explain why this area has not seen more widespread implementation of plasma level monitoring. The volume edited by Burroughs and Norman, however, is a significant contribution to the literature on this topic. It is a comprehensive volume dealing with many aspects of drug monitoring in the psychiatric field. The initial chapter by Lang on the mechanism of action of psychotropic drugs, provides an interesting introduction to the pharmacology of these compounds. Two interesting chapters follow, on methods for the measurement of psychotropic drugs-antidepressants, antipsychotics and antianxiety agents-which deal comprehensively with all modern techniques. The chapter by Graham shows application of these techniques to the measurement of the pharmacokinetics of tricyclic antidepressants. This is followed by an excellent chapter by Burroughs and Norman dealing with the relationship between plasma levels and clinical response in the anti-depressant field. The authors conclude that no consistent relationship exists between plasma levels and clinical response for tricyclic anti-depressants and that routine monitoring of plasma levels is not warranted. This contrasts with the measurement of serum lithium levels dealt with by Annitto and Gershon where only benefit is derived from determining correct therapeutic dosage and prevention in toxicity. Excellent chapters also follow on antipsychotic agents, Curry dealing with chlorpromazine in considerable depth, Sakalus and Traficante reporting on fluphenazine, Crammer on thioridazine and Evans on butyrophenones. It must be said, however, that the overall conclusion from these indepth appraisals is that little relationship exists between efficacy and plasma or blood antipsychotic levels. Sedvall and Grimm contribute on sampling CSF and plasma as tools for obtaining biochemical and pharmacokinetic data in neuroleptic therapy. They suggest some relationship exists between chlorpromazine treatment and clinical effect, at least in the early phases of treatment. Fulton and others contribute extensively on the pharmacokinetic of benzodiazepines. Justifiably, diazepam is given pride of place but further sections deal with other individual benzodiazepine compounds. Sedatives and hypnotics are dealt with by Wade but again the conclusion is that plasma concentrations of hypnosedatives correlate poorly with their effects, both at dosages used clinically and in overdosage. Eadie demonstrates the value of anticonvulsant drug plasma levels but concludes that the patient's clinical state should always be the final criterion in management and that plasma anticonvulsant levels should be seen only as a means to an end. The book is admirably finished by an overview by Hollister who concludes that there is little doubt that monitoring plasma levels of lithium and of anticonvulsants has made such treatment both safer and more effective. However, he is less certain of the role of tricyclic antidepressant monitoring and argues that there is little to favour monitoring of plasma concentrations of antipsychotic drugs. Hollister concludes that anti-anxiety and hypnotic drugs, which generally have a large margin of safety and suitable clinical criteria of response, should not be monitored. I find this overall to be a remarkably good book that is thoroughly recommended. I found much of interest and much new information and the editors must be congratulated for the production of such a volume. I would imagine, however, that at 150 Swiss francs many potential customers will be frightened away.

PG JENNER

Prevention and Treatment of Depression. Thomas A Ban, René Gonzalez, Assen S Jablensky, Norman A Sartorius and Felix E Vartanian. (Pp 300; £19.50.) Baltimore, University Park Press. Distributor: MTP Press Ltd. Lancaster, 1981.

This is the proceedings of a conference on depression organised by WHO and held in Washington in June 1980. Despite its title its 31 chapters embrace most aspects of depressive illness-epidemiology, classification, symptomatology, the role of biological and psychological factors in aetiology, various forms of treatment and models of care delivery from various parts of the world. The proceedings of confer- 